\title{
Verification of blackbodies emissivity
}

\author{
P. Nemeček ${ }^{\star}$ and I. Christov ${ }^{\star \star}$ \\ Slovak Institute of Metrology
}

Received: 31 May 2010 / Accepted: 19 August 2010

\begin{abstract}
Blackbody models are used as referential temperature measuring gauges for radiation thermometers calibration. Their emissivity is usually determined by calculation. The presented paper describes an experimental device used to measure local effective emissivities, using an optical radiometer. The methods to measure and evaluate emissivity and temperature are described. The results are compared with the theoretical model of a cylindrical-conical cavity used to calibrate radiation thermometers.
\end{abstract}

Keywords: English; CAFMET2010; papers, emissivity of blackbodies

\section{Introduction}

Blackbody models belong to the fundamental means for creating the temperature scales intended for the calibration of non-contacting thermometric devices. Typically, they are used as referential sources for the calibration of pyrometers at calibration laboratories. At this level, such laboratories use commercially produced blackbody models, whose characteristic variables - like local effective emissivity and local temperature - are frequently provided only as orientational values (e.g. as their minimum or maximum values), without uncertainties and mostly without any other parameters, e.g. wavelength that may parameterise the variables mentioned. Figure 1 illustrates how important the exactness of emissivity values is for the determination of temperatures with pyrometers functioning in two spectral ranges.

\section{Emissivity model of the technical realization of a blackbody}

The basic variables, which characterise a blackbody model, are temperature and emissivity. In contradiction to physical blackbody models, these variables relate to the cavity geometry and the emissivity characteristics of the material that covers the inner surface of the cavity alone. The principal source for differences between an ideal and the technical blackbody model is the opening through which radiation escapes. Thus the adiabaticity prerequisite has been challenged. The above mentioned differences resulting from the existing opening will be explicitly manifested by an anisotropic distribution of the radiation within the cavity and thus in the distribution of

* Correspondence: nemecek@smu.gov.sk

${ }^{\star \star}$ Correspondence: metrodat@netax.sk

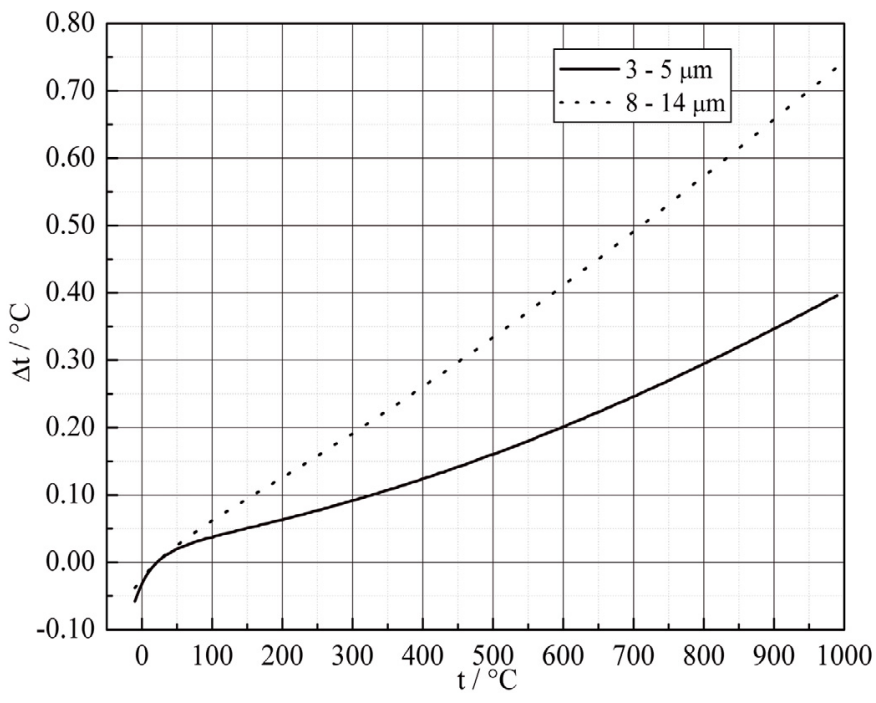

Fig. 1. The temperature error $\Delta t$ corresponding to a $0.1 \%$ change in the emissivity as measured using a pyrometer working in the spectral range of $3-5 \mu \mathrm{m}$ or $8-14 \mu \mathrm{m}$ at $21.5^{\circ} \mathrm{C}$.

temperatures throughout the cavity surface. Meanwhile the technical execution used to heat the cavity interior, any compensation of the influence of the irregular intensity of irradiation of the cavity surface due to the radiation escape opening is in fact impossible. We may describe the influence of the cavity geometry and of the emissivity of the material alone as follows:

1. intrinsic local effective emissivity $\varepsilon_{a i}{ }^{6}(x, y, z, \theta, \varphi, \lambda$, $\left.T_{i}\right)$, where $x, y$, and $z$ stand for common Cartesian co-ordinates of the cavity surface, and $\theta$ and $\varphi$ stand for angular coordinates characterising the direction of radiation; wavelength $\lambda$ and temperature $T_{i}$ parameterise the dependence of emissivity on these variables; 


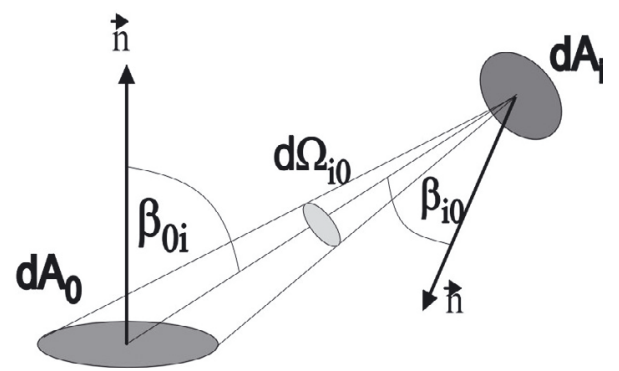

Fig. 2. Geometrical configuration factor.

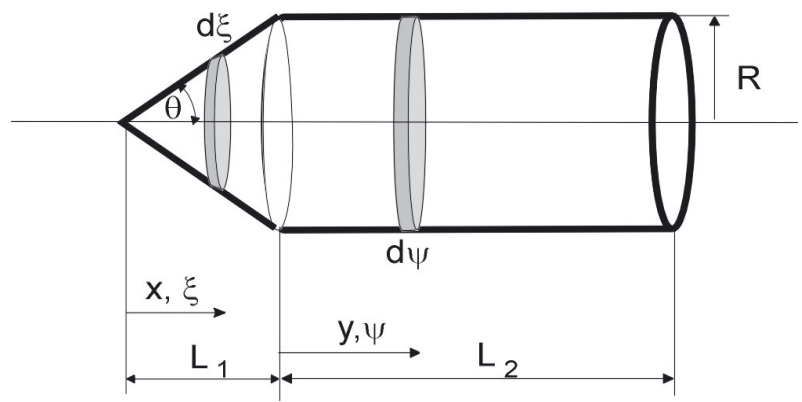

Fig. 3. The geometry of a cylindrical-conical cavity.

2. extrinsic local effective emissivity $\varepsilon_{a e}(x, y, z, \theta, \varphi, \rho$, $E(C)$ ), where $\rho$ stands for reflectivity and $E(C)$ stands for the intensity of irradiation in a given point from the entire cavity.

Regarding that extrinsic emissivity is determined by irradiation intensity $E(C)$ and the latter results from local emissivity values throughout the entire cavity surface (indicated by $C$ ), an explicit analytical solution is principally impossible and therefore iteration methods are applied. As far as we suppose that the cavity surface - for a given temperature and a given wavelength or a given wavelength range - showing Lambertian behaviour is isothermal and its own emissivity $\varepsilon$ remains constant throughout the entire cavity surface and that we may neglect the radiation penetrating into the cavity from the ambient environment and being reflected by it, the resulting local emissivity $\varepsilon_{a 0}$ in point 0 shall result from the following equation:

$$
\begin{aligned}
\varepsilon_{a 0} & =\varepsilon+(1-\varepsilon) \cdot \int_{C} \frac{\varepsilon_{a i} \cdot \cos \beta_{0 i} \cdot d \Omega_{0 i}}{\pi} \\
& =\varepsilon+(1-\varepsilon) \cdot \int_{C} \varepsilon_{a i} d^{2} G_{0 i},
\end{aligned}
$$

see Figure 2 and $d^{2} G_{0 i}$ so-called elementary configuration factor.

To make the following calculations, we have followed the method developed by Bedford and Ma, which appeared in [1]. (Alas, due to failures to print subscripts, superscripts, and plus/minus signs, the original work [2] has been almost unusable.) In such mathematical model of cavities with circular symmetry, the differential of the geometrical configuration factor $d^{2} G$ along the axial coordinate has been replaced with a difference that is equal

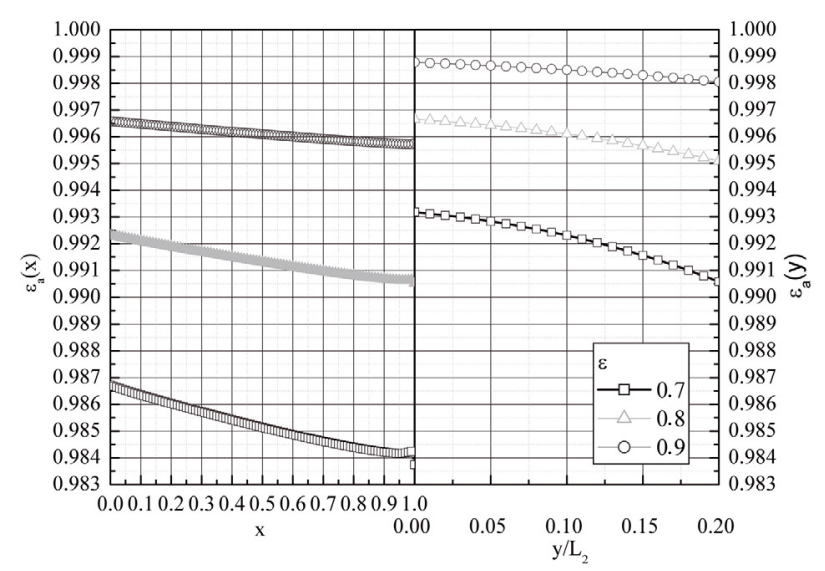

Fig. 4. The effective local emissivity values of an isothermal cylindrical-conical cavity surface with $L_{2}=8, \theta=60^{\circ}$, and $\varepsilon=0.7 ; 0.8 ; 0.9$.

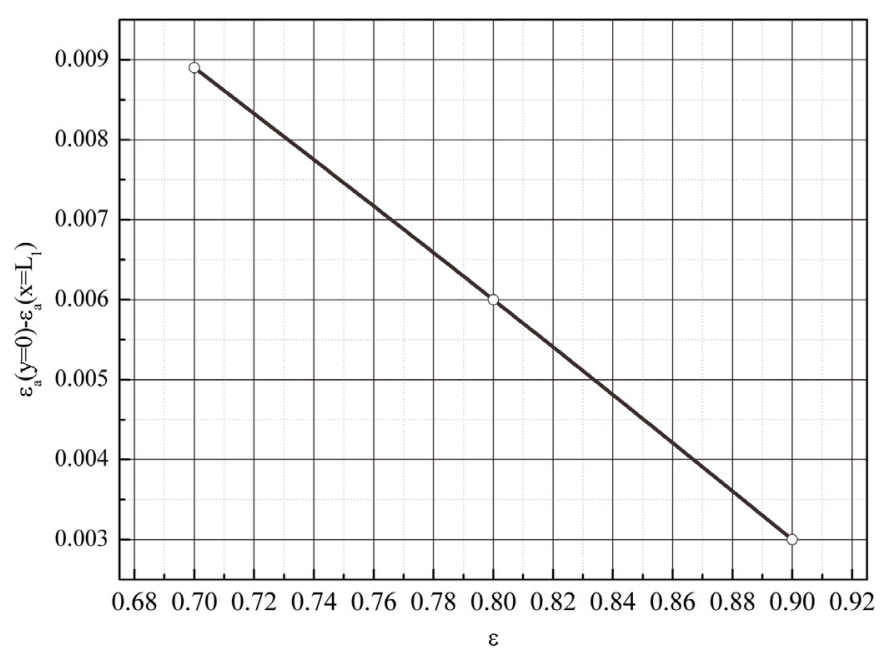

Fig. 5. Dependence of the local emissivity on the surface emissivity of an isothermal cylindrical-conical cavity with $L_{2}=8$, $\theta=60^{\circ}$.

to the difference between the configuration factors $d G$ of two adjacent circular discs. Thus the integral along the axial coordinate $x$ has been replaced with the aggregate of the differences of the differential configuration factors weighted with the local emissivity mean value. For example, the integral section of equation (1) for a conical cavity shall read:

$$
\begin{aligned}
\int_{0}^{L} \varepsilon_{a}(\xi) d^{2} G\left(x_{0}, \xi\right) & =\sum_{i=1}^{n} \frac{1}{2}\left[\varepsilon_{a}\left(\xi_{i+1}\right)+\varepsilon_{a}\left(\xi_{i}\right)\right] \\
& \times\left[\left|d G\left(x_{0}, \xi_{i+1}\right)-d G\left(x_{0}, \xi_{i}\right)\right|\right]
\end{aligned}
$$

where $\xi$ stands for the variable co-ordinate that discreetly changes with changing $i$ index, and $x_{0}$ stands for the coordinate of the point for which effective emissivity is determined.

The calculation of local effective emissivities emanates from the geometry of a cylindrical-conical cavity with the radius $(R)$ of $32.5 \mathrm{~mm}$, apex angle $(2 \theta)$ of $120^{\circ}$, the axis 


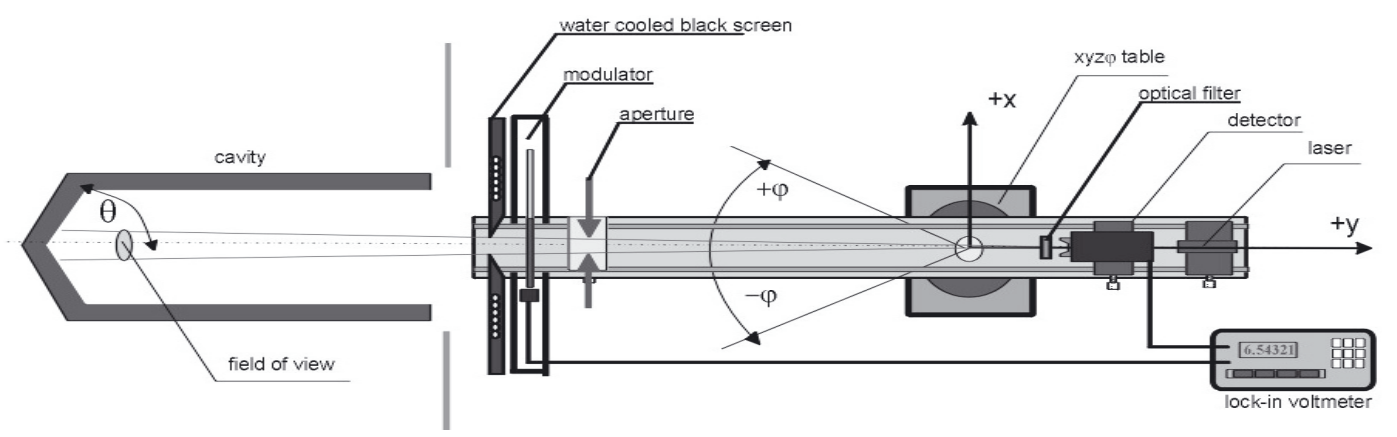

Fig. 6. A schematic diagram of the apparatus used to measure the local effective emissivity of the black body cavity.

segment $\left(L_{2}\right)$ of $160 \mathrm{~mm}$, and the own emissivity values of $0.7,0.8$ and 0.9 . We may suppose that the cavity surface emissivity may vary within the range of the three values mentioned above (Fig. 4).

The local effective emissivity courses provide a background for the determination of the cavity surface emissivity alone or for the verification and the improvement of its value. However, such theoretical models are bound to the prerequisites of surface isothermality and radiation isotropy. The isothermality prerequisite is expected to be fulfilled the best in point having its co-ordinates $x=L_{1}$ and $y=0$. In order to determine the emissivity we may use the dependence of the difference $\varepsilon_{a}(y \approx 0)-\varepsilon_{a}\left(x \approx L_{1}\right)$ on the emissivity values (Fig. 5).

\section{Experimental device}

The cavity radiance scanning at a given temperature and within a given spectral range is the principle, whose emissivity determination is based on. Non-selective RGT 18 pyroelectrical detector is the basic components of the experimental apparatus. A screen equipped with aperture mounted together with the detector, on a common basis, determines the field of vision of the device. The common basis also bears a cooled screen and a mechanical modulator. The axial alignment of the configuration whole and $f$ the blackbody cavity is secured by an adjustment laser device. The experimental apparatus has been set on a table that makes possible both rotation and lateral shifts $( \pm 40 \mathrm{~mm})$ of the apparatus along all the three axes. EG\&G 5208 lock-in voltmeter with synchronisation of phases from the mechanical modulator and working frequency of $15 \mathrm{~Hz}$ measures signals from the detector. The signals, together with the angle of rotation $\varphi$, are recorded in a PC. The optical filters selecting the spectral ranges of $8-14 \mu \mathrm{m}$ and of $3-5 \mu \mathrm{m}$ can be transferred to the input aperture of the detector.

\section{The measurement results and the estimate of measurement error sources}

Figure 6 indicates the results of the scanning of the blackbody model cavity with a temperature of $773 \mathrm{~K}$ and

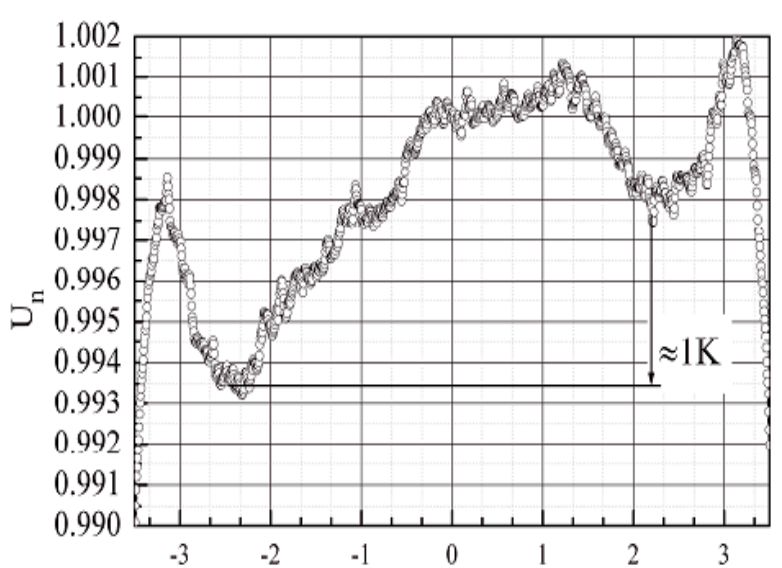

(A)

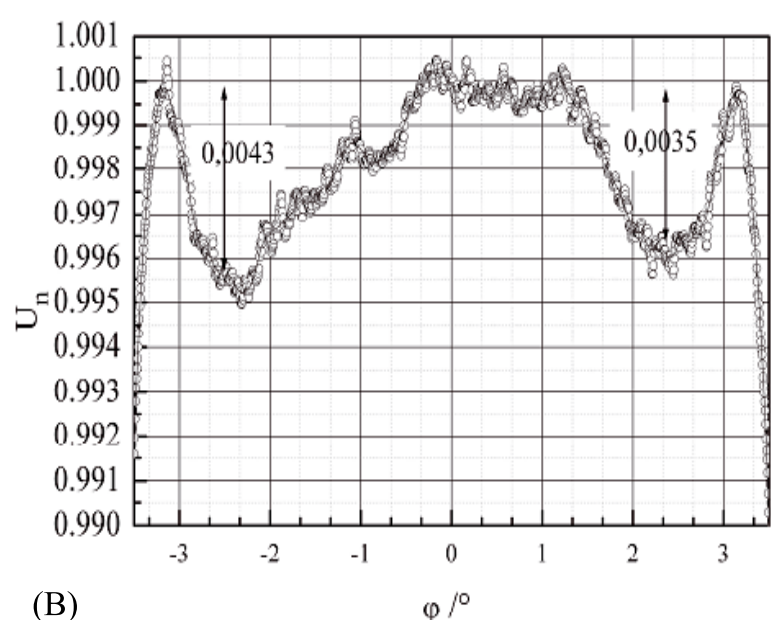

Fig. 7. (A) Normalised signal used to scan the blackbody. (B) The signal after correction on the temperature gradient.

the geometrical parameters indicated above. The scanning speed was $0.005^{\circ} \mathrm{s}$, with the time constant of the voltmeter $\tau=3 \mathrm{~s}$, and the period of entry of $3 \mathrm{~s}$.

The angular resolution used was $0.3^{\circ}$; this resolution corresponded to the diameters of $6 \mathrm{~mm}$ on the cavity bottom level and to the diameter of an ellipse $45 \mathrm{~mm}$ on the cylindrical wall. A pyroelectrical detector with window KRS-5 was used as the measuring device. The asymmetric 


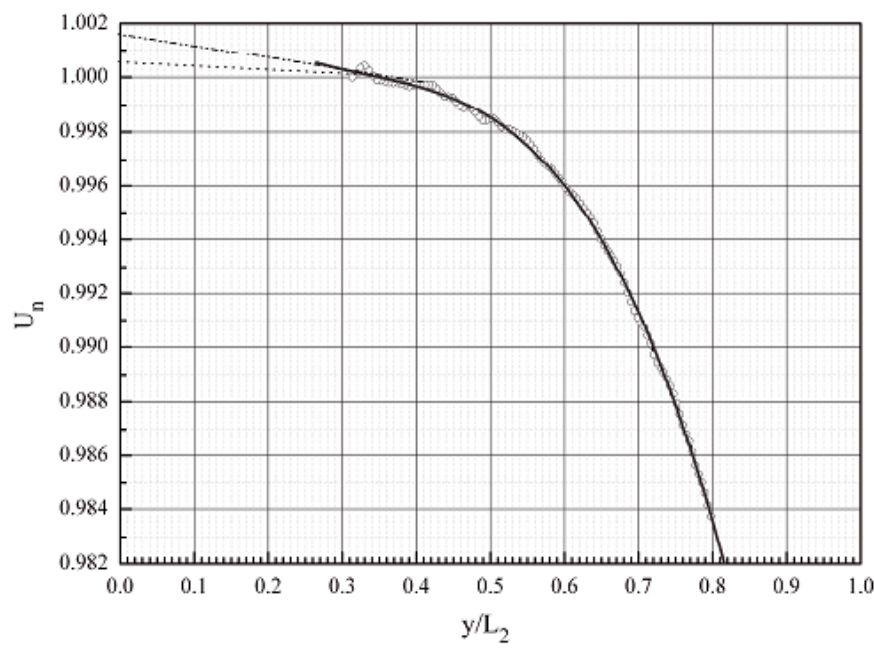

Fig. 8. Aproximation of local effective emissivity on the cylindrical wall.

radiation intensity profile corresponds to a radial temperature gradient of $1 \mathrm{~K}$ on the cavity bottom.

From the difference in the values measured we can obtain the fundamental difference $\Delta \varepsilon_{1}=0.0039$ between the local emissivities of the cylindrical wall and the conical wall. This value corresponds to the difference between the mean radiance of the cylindrical wall at $100 \mathrm{~mm}$ length and the radiance of the conical wall near the joint between the cone section and the cylinder section of the cavity and it presents the mean of the both values. The calculation of the mean limits errors arising from the voltmeter time constant. To obtain the value of the local emissivity $\varepsilon_{a}(y \approx 0)$, we can extrapolate from the values measured (Fig. 8).

The values are interpolated with the 3rd degree polynomial. Their extrapolation, using the first derivative in point $y / L_{2}=0.33$ for the value $y / L_{2}=0$, can be considered the maximum value. In the way of analogy, the application of the combination of the first and the second derivations in point 0.33 for the value 0 can be considered a value close to the minimum which is identical with the value in point 0.33 .

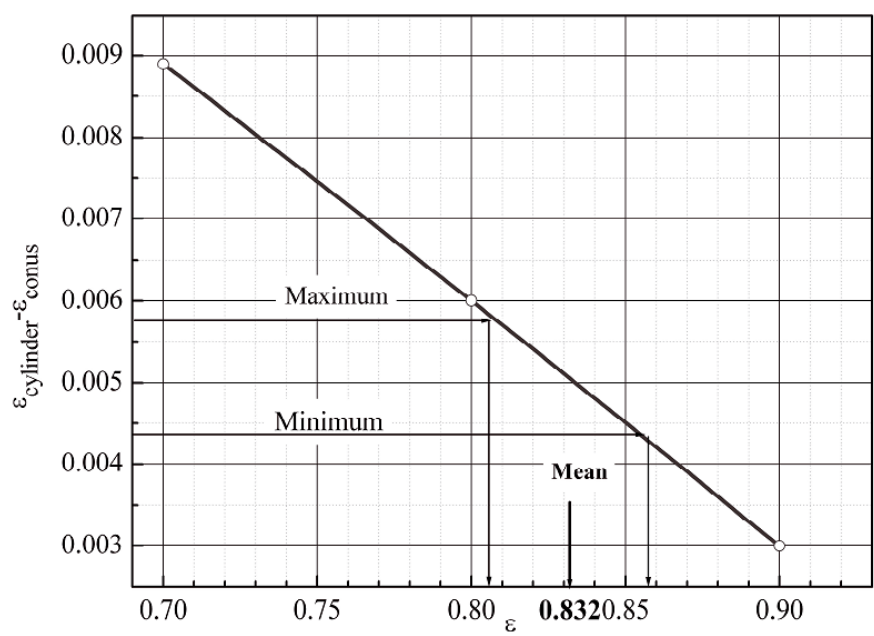

Fig. 9. The determination of material emissivity $(\square=0.832)$ from the measured difference of local effective emissivity. Maximum and minimum are the limits for this value.

\section{Conclusion}

The presented method of determining the own emissivity of the blackbody material is applicable in those cases, where we do not have sufficient information about the own emissivity, e.g. about its values in different spectral bands, or about its changes during the temperature variation. Therefore, it allows for a more precise temperature scale.

\section{References}

1. R.E. Bedford, C.K. Ma, Emissivities of diffuse cavities, II Isothermal and nonisothermal cylindro-cones, J. Opt. Soc. Am. 65, 565-572 (1975)

2. D.P. DeWitt, G.D. Nutter, Theory and Practice of Radiation Thermometry (John Wiley \& Sons, New York, 1988), p. 1138 